\section{Clinical practice recommendation on hematopoietic stem cell transplantation for acute myeloid leukemia patients with FLT3- internal tandem duplication: a position state- ment from the Acute Leukemia Working Party of the European Society for Blood and Marrow Transplantation}

Ali Bazarbachi ${ }^{12}$ Gesine Bug ${ }^{3}$ Frederic Baron ${ }^{4}$ Eolia Brissot, ${ }^{5}$ Fabio Ciceri, ${ }^{6}$ Iman Abou Dalle, ${ }^{1}$ Hartmut Döhner, ${ }^{7}$ Jordi Esteve, ${ }^{8}$ Yngvar Floisand, ${ }^{9,10}$ Sebastian Giebel, ${ }^{11}$ Maria Gilleece, ${ }^{12}$ Norbert-Claude Gorin, ${ }^{13}$ Elias Jabbour, ${ }^{14}$ Mahmoud Aljurf, ${ }^{15}$ Hagop Kantarjian, ${ }^{14}$ Mohamed Kharfan-Dabaja, ${ }^{16}$ Myriam Labopin, ${ }^{17,5}$ Francesco Lanza, ${ }^{18}$ Florent Malard, ${ }^{5}$ Zinaida Peric, ${ }^{19}$ Thomas Prebet, ${ }^{20}$ Farhad Ravandi, ${ }^{14}$ Annalisa Ruggeri, ${ }^{21}$ Jaime Sanz, ${ }^{22}$ Christoph Schmid, ${ }^{23}$ Roni Shouval, ${ }^{24}$ Alexandros Spyridonidis, ${ }^{25}$ Jurjen Versluis, ${ }^{26}$ Norbert Vey. ${ }^{27}$ Bipin N Savani, ${ }^{28}$ Arnon Nagler $^{29}$ and Mohamad Mohty

${ }^{1}$ Bone Marrow Transplantation Program, Department of Internal Medicine, American University of Beirut Medical Center, Beirut, Lebanon; '2Department of Anatomy, Cell Biology, and Physiological Sciences, American University of Beirut, Beirut, Lebanon; ${ }^{3}$ Department of Medicine 2, Hematology and Oncology, University Hospital, Goethe University Frankfurt, Frankfurt am Main, Germany; ${ }^{4} \mathrm{GIGA}-13$, University and CHU of Liège, Liège, Belgium; ${ }^{5}$ Sorbonne Universités, UPMC University of Paris 06, INSERM, Centre de Recherche SaintAntoine (CRSA), Hematology Department, AP-HP, Saint Antoine Hospital, Paris, France; ${ }^{6}$ Vita-Salute San Raffaele University of Milan, Milan, Italy; Hematology and Bone Marrow Transplantation Unit, IRCCS San Raffaele Scientific Institute, Milan, Italy; 'Department of Internal Medicine III, Ulm University Hospital, Ulm, Germany; ${ }^{8}$ Hematology Department, Hospital Clínic of Barcelona, IDIBAPS, University of Barcelona, Barcellona, Spain; 9Department of Hematology, Oslo University Hospital - Rikshospitalet, Oslo, Norway; ${ }^{10}$ Center for Cancer Cell Reprogramming, Institute for Cancer Research, Oslo University Hospital, Montebello, Oslo, Norway; ${ }^{11}$ Department of Bone Marrow Transplantation and Oncohematology, Maria Sklodowska-Curie Institute - Oncology Center, Gliwice Branch, Gliwice, Poland; ${ }^{12}$ Department of Haematology, Leeds Teaching Hospitals Trust, Leeds, UK; ${ }^{13}$ Department of Hematology and Cell Therapy, European Society for Blood and Marrow Transplantation, Paris Office, Hopital Saint-Antoine, Paris, France; ${ }^{14}$ Department of Leukemia, The University of Texas MD Anderson Cancer Center, Houston, TX, USA; ${ }^{15}$ Department of Hematology King Faisal Specialist Hospital \& Research Center, Riyadh, Saudi Arabia; ${ }^{16}$ Division of Hematology-Oncology and Blood and Marrow Transplantation and Cellular Therapies Program, Mayo Clinic, Jacksonville, FL, USA; ${ }^{17}$ Acute Leukemia Working Party, Paris Study Office, European Society for Blood and Marrow Transplantation, Paris, France; ${ }^{18}$ Romagna Transplant Network, Ravenna, Italy; ${ }^{19}$ University Hospital Center Zagreb, School of Medicine, University of Zagreb, Zagreb, Croatia; ${ }^{20}$ Section of Hematology, Department of Internal Medicine, Yale University School of Medicine, New Haven, CT, USA; ${ }^{21}$ Department of Pediatric Hematology and Oncology, IRCCS Bambino Gesù Children's Hospital, Roma, Italy; Eurocord, Hôpital Saint Louis, Paris, France; ${ }^{22}$ Hematology Department, Hospital Universitari i Politecnic La Fe. Instituto de Investigación Sanitaria La Fe, Valencia, CIBERONC, Instituto Carlos III, Madrid, Spain; ${ }^{23}$ Department of Hematology and Oncology, Augsburg University Hospital, Augsburg, Germany; ${ }^{24}$ Adult Bone Marrow Transplantation Service, Memorial Sloan Kettering Cancer Center, New York, NY, USA; ${ }^{25} \mathrm{BMT}$ Unit, University of Patras, Patras, Greece; ${ }^{26}$ Erasmus University Medical Center Cancer Institute, Rotterdam, the Netherlands; ${ }^{27}$ Department of Hematology, Institut PaoliCalmettes, Marseille, France; ${ }^{28}$ Department of Hematology-Oncology, Vanderbilt University Medical Center, Nashville, TN, USA and ${ }^{29} \mathrm{Hematology}$ and Bone Marrow Transplantation Division, Chaim Sheba Medical Center, Tel-Hashomer, Sackler School of Medicine, Tel Aviv University, Israel

\section{ABSTRACT}

T The FMS-like tyrosine kinase 3 (FLT3) gene is mutated in $25-30 \%$ of patients with acute myeloid leukemia (AML). Because of the poor prognosis associated with FLT3-internal tandem duplication mutated AML, allogeneic hematopoietic stem-cell transplantation (SCT) was commonly performed in first complete remission. Remarkable progress
Ferrata Storti Foundation

Haematologica 2020

Volume 105(6):1507-1516

\section{Correspondence:}

ALI BAZARBACHI,

bazarbac@aub.edu.lb

Received: November 19, 2019.

Accepted: March 19, 2020.

Pre-published: April 2, 2020

doi:10.3324/haematol.2019.243410

Check the online version for the most updated information on this article, online supplements, and information on authorship \& disclosures: www.haematologica.org/content/105/6/1507

(C)2020 Ferrata Storti Foundation

Material published in Haematologica is covered by copyright. All rights are reserved to the Ferrata Storti Foundation. Use of published material is allowed under the following terms and conditions:

https://creativecommons.org/licenses/by-nc/4.0/legalcode. Copies of published material are allowed for personal or internal use. Sharing published material for non-commercial purposes is subject to the following conditions:

https://creativecommons. org/licenses/by-nc/4.0/legalcode, sect. 3. Reproducing and sharing published material for commercial purposes is not allowed without permission in writing from the publisher. 
has been made in frontline treatments with the incorporation of FLT3 inhibitors and the development of highly sensitive minimal/measurable residual disease assays. Similarly, recent progress in allogeneic hematopoietic SCT includes improvement of transplant techniques, the use of haplo-identical donors in patients lacking an HLA matched donor, and the introduction of FLT3 inhibitors as post-transplant maintenance therapy. Nevertheless, current transplant strategies vary between centers and differ in terms of transplant indications based on the internal tandem duplication allelic ratio and concomitant nucleophosmin-1 mutation, as well as in terms of post-transplant maintenance/consolidation. This review generated by international leukemia or transplant experts, mostly from the European Society for Blood and Marrow Transplantation, attempts to develop a position statement on best approaches for allogeneic hematopoietic SCT for AML with FLT3-internal tandem duplication including indications for and modalities of such transplants and on the potential optimization of post-transplant maintenance with FLT inhibitors.

\section{Introduction}

FMS-like tyrosine kinase 3 (FLT3) is a transmembrane ligand-activated receptor tyrosine kinase that is normally expressed by hematopoietic stem cells and early myeloid and lymphoid progenitor cells, and is involved in the proliferation, differentiation and apoptosis of hematopoietic cells ${ }^{1}$ through various signaling pathways, including phosphatidylinositol 3-kinase (PI3K) and rat sarcoma (RAS) signal-transduction cascades..$^{2-7}$ FLT3 is mutated in about 25 $30 \%$ of newly diagnosed cases of acute myeloid leukemia (AML), ${ }^{8-10}$ either by internal tandem duplications (FLT3ITD) of the juxtamembrane domain (19-25\%), and/or by a point mutation, usually involving the tyrosine kinase domain (TKD) at D835 or I836 in the activating loop (7$10 \%) .{ }^{11-13}$ Both mutations are more frequent in cytogenetically normal AML and both constitutively activate FLT3 causing dimerization in a ligand-independent manner, resulting in proliferation and survival of leukemia cells. ${ }^{14,15}$

FLT3-ITD mutations in newly diagnosed AML are associated with a greater disease burden, manifesting as an elevated white blood cell count and a high percentage of blasts at the time of diagnosis as well as a tendency to early relapse and a poor overall prognosis., , $10-12,16,17$ Both European LeukemiaNet (ELN) recommendations and National Comprehensive Cancer Network (NCCN) guidelines incorporate FLT3-ITD mutations in risk-stratifying patients based on allelic burden and nucleophosmin-1 (NPM1) co-mutation. ${ }^{18,19}$ In cytogenetically normal patients, FLT3-ITD mutations in the presence of a concomitant NPM1 mutation, mainly when the FLT3-ITD allele ratio is low $(<0.5)$, fare better than those with wildtype NPM1. 8,10,16,17,20-22 Despite the great effort to harmonize and cross-validate the FLT3 assays within clinical trials, ${ }^{23}$ there is still no consensus on the FLT3-ITD allele ratio threshold and there is considerable variability between centers in the assessment of the FLT3-ITD ratio according to the technique used, if one is available. Furthermore, in addition to NPM1 mutations, a significant overlap with other mutations (WT1, IDH1, DNMT3A) as well as NUP98/NSD1 fusions modify outcome as well as response to therapy. Although patients with FLT3-ITD AML respond to conventional induction chemotherapy with remission rates similar to those seen in other subtypes of AML, they are much more likely to relapse and to relapse quickly. ${ }^{11,12,24-28}$ The prognostic impact of FLT3-TKD is less clear, ${ }^{29-32}$ but it, too, is influenced substantially by NPM1 co-mutation which confers a better prognosis. ${ }^{33-35}$
The availability of active FLT3 inhibitors that are able to disrupt the oncogenic signaling initiated by FLT3 has improved the overall survival (OS) of patients with FLT3mutated AML. ${ }^{36}$ Midostaurin, a multikinase inhibitor, was granted Food and Drug Administration (FDA) and European Medicines Agency (EMA) approval for the treatment of patients with newly diagnosed FLT3-mutated AML, in combination with intensive chemotherapy, and by the EMA in addition as maintenance treatment after conventional consolidation therapy. This approval was based on the results of the RATIFY trial, which demonstrated that the combination of midostaurin with standard induction therapy resulted in significantly prolonged OS (not censored for transplant) for AML with either FLT3ITD or FLT3-TKD mutations. ${ }^{37}$ The benefit was particularly remarkable in patients who went on to receive allogeneic hematopoietic stem cell transplantation (allo-SCT) in first complete remission (CR1). Following the results of the ADMIRAL trial, gilteritinib, a second-generation FLT3 inhibitor, was recently approved for relapsed/refractory FLT3-mutated AML with FLT3-ITD and FLT3-TKD mutations. ${ }^{38}$ Promising data were also reported for quizartinib and crenolanib..$^{99,40}$ Finally, because of its long-time availability, sorafenib has been tested, alone or in combination, in various settings in FLT3-ITD AML, such as first-line therapy $^{41,42}$ or for the treatment of disease relapse, ${ }^{43-45}$ including after failure of allo-SCT. ${ }^{45-57}$ However, recent data appear to support incorporating sorafenib into the treatment of patients with FLT3-mutated AML, possibly with induction therapy ${ }^{41,58,59}$ as well as maintenance therapy after allo-SCT. ${ }^{43,60-65}$

Because of the diversity in FLT3-mutated AML, which depends on the type of FLT3 mutation, FLT3-ITD allelic burden, insertion site and co-occurring mutations, the decision regarding whether to perform allo-SCT in CR1 is becoming more challenging. ${ }^{66-75}$ With the use of more effective therapies, especially with the incorporation of FLT3 inhibitors, deeper responses are being achieved. The assessment of minimal/measurable residual disease $(\mathrm{MRD})$ at the time of response has enabled prediction of outcomes in AML, and tailoring of post-remission therapeutic strategies accordingly. ${ }^{76-78}$ Additionally, substantial progress has been made in allo-SCT in recent years, including improvement of transplant techniques, the use of haplo-identical donors in patients lacking an HLAmatched donor, ${ }^{79-81}$ and post-transplant preventive strategies, such as prophylactic or preemptive use of FLT3 inhibitors. ${ }^{63,82-85}$ Nevertheless, current transplant strategies 
vary between centers and differ in terms of indications for the transplants and treatments following them. This review provides a consensus from European Society for Blood and Marrow Transplantation (EBMT) experts on best approaches to allo-SCT in AML with FLT3-ITD including the indications for and modalities of allo-SCT and on potential optimization of post-transplant maintenance therapy with FLT3 inhibitors.

\section{The consensus process}

Two chairpersons (AB and $M M$ ) appointed a panel of 32 physicians (hereafter referred to as the Panel) selected mostly from the EBMT) for their expertise in research and clinical practice in AML and allo-SCT. A physician with expertise in clinical epidemiology (ML) ensured the methodological correctness of the process. The objective of the Panel was to identify practical issues pertinent to all physicians involved in the therapeutic management of patients undergoing allo-SCT for AML with FLT3 mutations and to generate best practice recommendations on indications for and modalities of allo-SCT and on potential optimization of post-transplant maintenance with FLT3 inhibitors. This was done through a number of questions according to the Delphi technique. ${ }^{86}$ A search for relevant literature in English was performed in the MEDLINE, EMBASE and PubMed databases (up to August 2019). Most of the studies used for these recommendations are retrospective cohort studies or phase II trials, with only a few prospective randomized trials. Three panelists drafted statements that addressed the key questions identified, and the remaining panelists scored their agreement with those statements and provided suggestions for rephrasing them.

The evaluation of evidence and the subsequent recommendations were graded according to the system used by Couriel. ${ }^{87}$ The strength of the recommendations (Online Supplementary Table S1) and evidence levels (Online Supplementary Table S2) were rated by all participants of the consensus process.

\section{Overview of prognosis and current indications for allogeneic stem-cell transplantation in FLT3-mutated acute myeloid leukemia}

The indication for allo-SCT in FLT3-ITD AML depends largely on FLT3 variables (allelic burden, insertion site and co-occurring mutations), on disease status (including $\mathrm{MRD}$ ), and on the use of FLT3 inhibitors during induction/consolidation treatment, in addition to other patient-, donor- and graft-related factors. Unfortunately, there are no prospective randomized trials evaluating the best post-remission therapeutic strategy in FLT3-mutated AML, taking in consideration all the diverse combinations.

Several recent reports have suggested that allele burden might affect prognosis of FLT3-ITD AML treated with standard induction chemotherapy. ${ }^{17,22,88,89}$ Indeed, the presence of a high allelic burden of FLT3-ITD mutations $(\geq 0.5)$ confers a poor prognosis. ${ }^{12,27,90,91}$ Several studies have demonstrated that allo-SCT significantly improves survival outcomes in this category ${ }^{69,92-95}$ and that the negative impact of high allele burden might be overcome when patients undergo allo-SCT in CR1. ${ }^{17}$ Therefore, all patients with FLT3-ITD high should be considered for allo-SCT in CR1. ${ }^{66,69,92-96}$ These patients still face higher rates of early relapse and poor responses to further therapy and eventually poor long-term survival. ${ }^{92,97}$ The worst prognosis is observed in patients who relapse after allo-SCT, who have predicted 1-year OS rates below 20\%. ${ }^{98}$ However, a subcategory of patients with FLT3-ITD ${ }^{\text {high }} /$ NPM1 mutation of the ELN intermediate-risk group treated with FLT3 inhibitors, and who achieve MRD negativity, may be offered the possibility of post-remission consolidation with longitudinal MRD monitoring of NPM1.91 This approach should be undertaken with caution, and preferably within a clinical trial, since recent data suggest the possible extinction of the NPM1 clone after chemotherapy while the FLT3-ITD clone persists.

Additional mutations may, however, influence the prognosis of AML with FLT3-ITD. For example, the co-existence of NPM1 mutation with FLT3-ITD is associated with improved outcomes, particularly in patients with a low FLT3 allelic ratio $(<0.5) .8,10,16,20$ According to the 2017 ELN recommendations, this subcategory is stratified as favorable risk, advocating against the need for allo-SCT. ${ }^{91}$ Nonetheless, the good prognosis of a low allelic ratio is not universally recognized, with data suggesting better outcome for allografted patients regardless of NPM1 mutation status. ${ }^{99}$ A threshold for FLT3 allelic burden is also controversial and differs according to studies. It was mainly based on the median of the mutant-to-wildtype ratio found in different retrospective studies. For example, in one study evaluating the prognostic factors of newly diagnosed AML, a FLT3 ratio above 0.78 was associated with worse survival, whereas in another study the threshold was $0.51 .^{11,17}$ Therefore, the allelic burden has a continuous effect on survival outcomes and a ratio of 0.5 is a chosen threshold based on maximum clinical prognostic data. With the advent of FLT3 inhibitors in the frontline treatment of FLT3-mutated AML, the OS has improved regardless of the allelic burden and the use of allo-SCT. Whether NPM1-mutant FLT3-ITD ${ }^{\text {low }}$ AML warrants postremission allo-SCT in CR1 or not is still debatable. Although some studies analyzing the effect of allo-SCT in patients with NPM1-mutant FLT3-ITD ${ }^{\text {low }}$ found no improvement in OS or relapse risk, we must take into consideration the retrospective nature of the analysis and the small number of patients with a non-statistically significant improvement in OS and relapse risk. ${ }^{17,22}$ Interestingly, patients with newly diagnosed AML with NPM1-mutant FLT3-ITD ${ }^{\text {low }}$ treated with frontline midostaurin and intensive chemotherapy, had a 3 -year OS rate of around $75 \%$. In a retrospective subgroup analysis, the benefit of alloSCT was only seen in the adverse ELN subgroup [hazard ratio $(\mathrm{HR})=0.39 ; P=0.003]$, but not in the favorable $(\mathrm{HR}=0.78 ; P=0.62)$ and intermediate risk subgroups $(\mathrm{HR}=0.81 ; P=0.53) .{ }^{91}$ These findings should, however, be interpreted with caution as the RATIFY trial was not powered to demonstrate a difference of benefit of allo-SCT among diverse FLT3-ITD/NPM1 genotypes. For example, the total number of patients in the favorable ELN subgroup was 85 and these patients were divided into four small groups according to whether they did or did not receive midostaurin and/or allo-SCT in CR1. ${ }^{91}$

The deleterious effect of FLT3-ITD was most clinically relevant in patients with concomitant NPM1 and DNMT3A mutations, suggesting that AML patients with NPM1, FLT3-ITD and DNMT3A mutations (triple-positive AML) should be transplanted regardless of the FLT3-ITD allelic ratio. ${ }^{8}$ A recent study conducted on 147 patients 
found that NPM1-positive AML with low allelic FLT3ITD still had an unfavorable outcome, with an OS rate of only $41 \%$, but with significant improvements in both relapse-free survival (RFS) and OS for those allografted in CR1. ${ }^{99}$ This challenges the notion of withholding transplant for patients with supposedly favorable outcomes. In that sense, a recent study from the $\mathrm{MD}$ Anderson Cancer Center showed that allo-SCT improved leukemia-free survival (LFS) and OS independently of the FLT3-ITD allelic ratio and NPM1 mutation status. ${ }^{100}$ This fits with recent NCCN guidelines still offering allo-SCT for all patients with FLT3-ITD mutations regardless of allelic ratio or NPM1 mutation status. ${ }^{18}$

On the other hand, patients with a low allelic ITD ratio lacking an NPM1 mutation (and lacking other adverse risk mutations) are currently considered intermediate risk, hence in a gray prognostic area with no proper consensus on optimal treatment strategy. There is conflict regarding the current practice between proceeding to allo-SCT for these patients or limiting allo-SCT only to those who do not achieve MRD negativity by multiparametric flow cytometry. Indeed, technical limitations prevent the use of FLT3 mutation for assessment of MRD which must therefore rely on multiparametric flow cytometry. ${ }^{101}$ Finally, Versluis et al. reported that in patients with wildtype NPM1 AML without FLT3-ITD or with a low allelic ratio of FLT3-ITD, reduced intensity conditioning allo-SCT resulted in better OS and RFS rates as compared with chemotherapy or autologous SCT. ${ }^{89}$

Overall, limitations to the universal incorporation of FLT3-ITD allelic ratio into routine clinical practice and the treatment algorithm include the lack of a clear cut-off $(0.5$ in the ELN recommendations, 0.7 in the RATIFY study) and the potential variability of the allelic ratio over time. A global effort is needed to standardize the technique for determining the FLT3-ITD allelic ratio, making it universal with calibration of all laboratories, reminiscent of the global exercise the world did for BCR/ABL1. Similarly, the definition of high and low allelic ratio should also be standardized with a clear consensus on a cut-off level. Until these technical challenges are addressed, the transplant indication remains controversial in patients with FLT3-ITD who belong to the ELN favorable risk group (low allelic ratio $<0.5$ with concomitant NPM1 mutation) and who achieve MRD negativity. Many European cooperative groups follow the ELN algorithm, deferring allo-SCT in patients with NPM1-mutant FLT3-ITD ${ }^{\text {low }}$, unless there is molecular persistence of NPM1. Thus, performing MRD assessment regularly to decide on allo-SCT timing is crucial when selecting this approach. Conversely, the NCCN guidelines are still advocating allo-SCT in CR1 in this setting.

Finally, data on the prognosis of FLT3-TKD AML remain conflicting, with some studies suggesting a negative impact of TKD mutations on LFS and OS, ${ }^{11,25,30}$ while others suggesting no prognostic effect, or even a benefit when a NPM1 mutation is present. ${ }^{29,32,34,35}$

\section{Hematopoietic stem cell transplantation and factors predictive of outcome}

As stated above, because of the poor prognosis associated with FLT3-ITD mutated AML, allo-SCT was most frequently performed in patients in CR1 $1^{66-74,102}$ including fit patients $\geq 60$ years of age. ${ }^{103}$ In most studies, the LFS rate at 2 years ranges between 50 to $60 \%$ in that setting, ${ }^{66,92,97,104}$ although a wide variation from $20 \%{ }^{70,105}$ to $70 \%{ }^{69}$ has been reported. There are knowledge gaps about the factors that can predict outcome after allo-SCT.

A previous EBMT study ${ }^{97}$ reported that patients with FLT3-ITD mutated AML with concomitant mutated NPM1 had better post-transplant outcomes compared to those with wildtype NPM1. Similarly, other studies reported that the presence of active disease or MRD before allo-SCT results in poor post-transplant outcomes. ${ }^{106,107}$

A recent, large EBMT registry study assessed outcomes in 462 allografted FLT3-mutated AML patients with a median follow-up of 39 months for alive patients. ${ }^{63}$ Forty percent received allo-SCT from matched related donors, $49 \%$ from matched unrelated donors and 11\% from haploidentical donors. Two-year cumulative incidence of relapse (CIR) and non-relapse mortality rates were $34 \%$ and $15 \%$, respectively, whereas LFS, OS and graft-versus-host disease (GvHD)-free. relapse-free survival (GRFS) rates were 51\%, $59 \%$ and $38 \%$, respectively. On multivariable analysis, the need for more than one induction treatment negatively affected outcome, while prescribing an allo-SCT in CR1 resulted in improved CIR, LFS and OS. Presence of an NPM1 mutation was also associated with better outcomes, including better CIR, LFS, OS and GRFS. Post-transplant maintenance therapy with sorafenib significantly reduced the CIR and improved LFS, OS and GFRS. Outcomes were not affected by the type of donor or conditioning intensity. An important finding from this study was that in vivo T-cell depletion with antithymocyte globulin decreased chronic GvHD and significantly improved LFS, OS and GRFS, without an apparent increase in the risk of relapse. This indicates that, even in the setting of FLT3-mutated AML, in vivo T-cell depletion does not appear to abrogate the graft-versusleukemia effect. Finally, the use of haplo-identical donors was associated with improved GRFS compared to that achieved with other types of donors. Given the high risk of rapid relapse of patients with FLT3-mutated AML in CR1 and the poor outcome of allo-SCT in CR2 or beyond, ${ }^{11,12,108}$ these results and those of a recent EBMT study suggest that, in the absence of a matched sibling donor, performing haplo-identical transplants in CR1 may be considered. ${ }^{109}$ Furthermore, in another large EBMT study on more than 6,500 adult AML patients allografted in CR1, multivariate analysis confirmed the lack of a statistically significant difference in OS following transplants from matched related donors or 10/10 matched unrelated donors, or haplo-SCT. ${ }^{110}$ Finally, the results of a CIBMTR, EUROCORD and EBMT collaborative analysis demonstrated that outcomes after umbilical cord blood transplantation are similar to those after allo-SCT from sibling donors for patients with FLT3ITD AML. ${ }^{110}$

\section{Post-transplant maintenance in FLT3-mutated acute myeloid leukemia}

Even after allo-SCT, FLT3-mutated AML is associated with a higher risk of early relapse (30\%-59\%) compared to FLT3-wildtype AML. ${ }^{82,92}$ Indeed, in a CIBMTR analysis of 511 patients (158 with FLT3 mutations), there was an increase in relapse rates in FLT3-mutated AML (38\% vs. $28 \% ; P=0.04$; relative risk $1.60 ; 95 \% \mathrm{CI}: 1.15-2.22) .{ }^{74}$ Satisfactory treatment of patients with FLT3-mutated AML who relapse or progress after allo-SCT, is an unmet need. Chemotherapy or FLT3 inhibitors alone or com- 
bined with donor lymphocyte infusions are rarely effective in the long term, ${ }^{45-50}$ even though a small proportion of patients who relapse after allo-SCT can achieve longlasting responses with sorafenib. ${ }^{52,54,55,57}$ A second allo-SCT can be offered to only a small percentage of patients and is associated with a rather high non-relapse mortality rate. ${ }^{111}$ Several studies have, therefore, investigated the use of post-transplant maintenance with FLT3 inhibitors as a strategy aimed to reduce relapse after allo-SCT. ${ }^{112}$

Midostaurin was not offered as maintenance therapy to recipients of allo-SCT in the RATIFY study, ${ }^{113}$ but the RADIUS phase II randomized trial compared post-transplant midostaurin maintenance with standard care in 60 adult patients. ${ }^{114}$ Estimated relapse rates at 18 months were $24 \%$ in the standard care group and $11 \%$ in the midostaurin group $(P=0.27) .{ }^{114}$ In another prospective phase II study, maintenance midostaurin was also offered to FLT3-mutated AML patients undergoing allo-SCT in CR1. In a landmark analysis in patients who were event free at day 100 after transplant $(n=116)$, those who started maintenance therapy within 100 days after their transplant $(n=72)$ had a significantly better OS than those who did not. ${ }^{115}$ The main cause of early discontinuation of maintenance midostaurin after allo-SCT (23\%) was poor tolerability, mainly as a result of gastrointestinal toxicity. ${ }^{114}$

Sorafenib has been studied as maintenance therapy following allo-SCT, demonstrating benefit with regards to survival and improved outcomes in a phase I study, a pilot study, a single-center study, a multicenter study, a registry study and a randomized study. ${ }^{60-65,116}$ A phase I trial (NCT01398501) was conducted in which 22 FLT3-ITD AML patients received twelve 28-day cycles of sorafenib 45-120 days after allo-SCT. ${ }^{61}$ The maximum tolerated dose was established at $400 \mathrm{mg}$ twice daily. The 1-year progression-free survival (PFS) rate was $85 \%$ with a corresponding 1 -year $\mathrm{OS}$ of $95 \%$. In a pilot study, six patients with FLT3-ITD AML received sorafenib ( $\mathrm{n}=5$ maintenance, $\mathrm{n}=1$ salvage) after allo-SCT with similarly encouraging results. ${ }^{116}$ Five of these patients developed cutaneous corticosteroid-sensitive GvHD within a few days after sorafenib initiation, suggesting a possible immunomodulatory effect, and remarkably all patients had sustained molecular remissions.

In a single-institution, observational study on FLT3-ITD AML patients transplanted in CR1, 26 patients who received sorafenib as maintenance treatment after alloSCT were compared to 55 historical controls who did not. ${ }^{62}$ The sorafenib cohort had a better 2 -year OS rate ( $81 \%$ vs. $62 \%$ ), improved PFS (82\% vs. $53 \%$ ), and lower relapse incidence ( $8 \%$ vs. $38 \%$ ).

In a multicenter study, 27 FLT3-mutated AML patients (aged 15-57 years) received maintenance therapy with sorafenib as a single agent after allo-SCT. ${ }^{60}$ At a median follow-up of 18 months, 25 patients were in complete remission with full donor chimerism, with 1-year PFS and OS rates reaching $92 \%$. Updated results after a median follow-up of 40 months further demonstrated favorable long-term outcomes in patients receiving sorafenib maintenance therapy, with 2-year PFS and OS rates reaching $73 \%$ and $80 \%$, respectively, with an acceptable toxicity profile. $^{65}$

A recent large EBMT registry study assessed outcomes in 462 allografted FLT3-mutated AML patients over a median follow-up of 39 months for surviving patients. ${ }^{63}$
Twenty-eight patients received post-transplant sorafenib maintenance treatment, initiated at a median of 55 days after transplantation (range, 1-173) at a median dose of $800 \mathrm{mg} /$ day (range, 200-800 mg/day). Thirteen patients in the sorafenib group had chronic GvHD at a median time of 76 days after the initiation of sorafenib (range, 9-194 days). Chronic GvHD was limited in seven patients and extensive in six. On multivariate analysis, post-transplant maintenance with sorafenib significantly reduced the relapse incidence $(\mathrm{HR}=0.39 ; P=0.05)$, and improved LFS $(\mathrm{HR}=0.35 ; P=0.01)$, OS $(\mathrm{HR}=0.36 ; P=0.03)$ and GFRS $(\mathrm{HR}=0.44 ; P=0.02)$. Matched-pair analysis was also performed on 52 patients (26 in the sorafenib group and 26 controls) who engrafted and survived after allo-SCT with no relapse or grade II-IV acute GvHD until sorafenib initiation. The 2 -year LFS and OS rates were $79 \%$ and $83 \%$, respectively, in the sorafenib group $(P=0.02)$ versus $54 \%$ and $62 \%$, respectively, in the controls $(P=0.007)$.

More recently, preliminary conclusions of a doubleblind, prospective trial (SORMAIN) that randomized patients to either maintenance treatment with sorafenib or placebo introduced during the first 60-100 days after allo-SCT provided further support for the use of this drug in this high-risk setting. ${ }^{64}$ Eighty transplanted FLT3-ITD adult AML patients were randomized 1:1 to receive either sorafenib (up to $400 \mathrm{mg}$ twice daily) or placebo for up to 24 months. After a median follow-up of 42 months, the median RFS was 31 months in the placebo group whereas it was 'not reached' in the sorafenib group (corresponding to a 2 -year RFS of $53 \%$ vs. $85 \%$ : HR $0.39 ; P=0.01$ ). Sorafenib was well-tolerated with toxicities that were generally manageable, mostly by dose reduction. These findings build on previously reported data and confirm that sorafenib maintenance therapy after allo-SCT in FLT3-ITD AML patients is both safe and efficient in significantly reducing CIR and improving survival.

In addition to sorafenib's direct anti-leukemic effect, a possible synergism between the drug and alloreactive donor $T$ cells in facilitating long-term disease control has been suggested, ${ }^{117}$ and has also been proposed in murine models in which sorafenib apparently exacerbated GvHD. ${ }^{118}$ A recent study demonstrated that sorafenib promotes graft-versus-leukemia activity in mice and humans through interleukin-15 production in FLT3-ITD leukemia cells. ${ }^{119}$

Gilteritinib is also currently being prospectively assessed for maintenance use in FLT3-ITD AML after alloSCT in a phase III, randomized, double-blind, placebocontrolled multicenter trial (NCT02997202). ${ }^{120}$ This study aims to enroll 346 adult patients with AML in CR1, randomized 1:1, to receive either gilteritinib $120 \mathrm{mg}$ or placebo for 2 years. In addition, a large phase III randomized study (NCT04027309) by a consortium of several cooperative study groups, including HOVON, AMLSG, SAKK, ALFA, CETLAM, PETHEMA, FILO and ALLG, is anticipated to start enrolling by the end of 2019: patients will be randomized to midostaurin or gilteritinib added to standard induction and consolidation treatment. Patients who achieve complete remission will continue maintenance with either midostaurin or gilteritinib.

Finally, the recent approval of midostaurin for frontline treatment of FLT3-mutated AML in the USA and Europe may challenge the role of post-transplant maintenance therapies, including sorafenib. Accordingly, new data should be generated in this setting. ${ }^{121,122}$ Most FLT3-mutat- 
ed AML patients, however, are not currently receiving midostaurin, at least outside the USA and some other countries; therefore, for the foreseeable future, patients may still benefit from sorafenib maintenance treatment after allo-SCT.

\section{Summary of position statement (Table 1)}

\section{1- Indications for allogeneic stem-cell transplantation in FLT3-internal tandem duplication acute myeloid leukemia}

- The indication for allo-SCT is controversial in patients with FLT3-ITD who belong to the ELN favorable risk group (low allelic ratio $<0.5$ with concomitant NPM1 mutation) and who achieve MRD negativity. Allo-SCT may be delayed until first relapse as recommended by the ELN or performed in CR1 as allowed by NCCN guidelines. Grade level C-II

- In general, all other patients with FLT3-ITD AML should be considered for allo-SCT in CR1 if feasible. Grade level B-II

\section{2- Modalities of hematopoietic stem cell transplantation}

- Donors should be selected according to EBMT general guidelines ${ }^{83}$ including the potential use of cord blood grafts whenever indicated. Grade level B-II

- In vivo T-cell depletion decreases the risk of chronic $\mathrm{GvHD}$, without apparently increasing the risk of relapse, in FLT3-ITD AML and is therefore an option in this setting. Grade level B-II

- The choice of conditioning has no direct link with FLT3-ITD mutation and should be adapted to other individual risk factors such as age, disease status at transplant, and donor type. Grade level B-II

\section{3- Post-transplant maintenance for FLT3-internal tan- dem duplication acute myeloid leukemia}

- Post-transplant maintenance therapy with a FLT3 inhibitor for patients who have undergone allo-SCT for FLT3-ITD AML is recommended (except for patients with active acute GvHD). In the absence of an appropriate clinical trial, sorafenib could be considered as the preferred option, but other FLT3 inhibitors are attractive and war-

Table 1. Summary of the European Society for Blood and Marrow Transplantation position statement on allogeneic hematopoietic stem-cell transplantation in FLT3-internal tandem duplication acute myeloid leukemia.

Indication for allo-SCT Transplant indication is controversial in patients with FLT3-ITD who belong to the ELN favorable risk group (low allelic

in FLT3 mutated AML ratio $<0.5$ with concomitant NPM1 mutation) and who achieve MRD negativity. Allo-SCT may be delayed until first relapse as recommended by the ELN or performed in CR1 as allowed by NCCN guidelines.

In general, all other patients with FLT3-ITD should be considered for allo-SCT in CR1 if feasible.

$\begin{array}{ll}\text { Modalities of allo-SCT } & \text { Donor selection according to EBMT general guidelines. } \\ \text { In vivo T-cell depletion decreases the risk of chronic GVHD without an apparent increase in the risk of relapse in FLT3 } \\ \text { mutated AML and is therefore an option in this setting. } \\ \text { The choice of conditioning has no direct link with FLT3 mutation and should be adapted to other individual risk factors } \\ \text { such as age, disease status at transplant, and donor type. }\end{array}$

Post-transplant maintenance

Post-transplant systemic maintenance therapy with a FLT3 inhibitor for patients who underwent allo-SCT for FLT3-ITD AML is recommended (except for patients with active acute GvHD).

In the absence of an appropriate clinical trial, sorafenib could be considered as the preferred option, but the role of other FLT3 inhibitors warrants investigation.

Maintenance treatment should be initiated as soon as possible after disease evaluation, including MRD assessment, especially in patients with MRD-positive AML before or after allo-SCT, provided there is adequate hematologic reconstitution.

The recommended post-transplant maintenance is sorafenib at a dose of $400 \mathrm{mg} /$ day in two divided doses. Patients with MRD-positive disease may receive $800 \mathrm{mg} /$ day in two divided doses, to be adapted according to tolerance. Sorafenib should be transiently discontinued in the case of GvHD requiring systemic treatment with corticosteroids, but may be cautiously resumed once remission of GvHD is documented.

Ongoing studies will determine whether midostaurin, gilteritinib or other FLT3 inhibitors will become additional alternatives in this setting.

Maintenance therapy duration is not firmly established, but a minimum of 2 years is recommended, depending on tolerance.

Allo-SCT: allogeneic hematopoietic stem cell transplantation; FLT3: FMS-like tyrosine kinase 3; AML: acute myeloid leukemia; FLT3-ITD: FLT3-internal tandem duplication; ELN European LeukemiaNet; NPM1: Nucleophosmin 1; MRD: minimal residual disease; CR1: first complete remission; NCCN: National Comprehensive Cancer Network; EBMT: European Society for Blood and Marrow Transplantation; GvHD: graft-versus-host disease. 
rant further investigation in larger prospective studies. Grade level B-II

- Maintenance therapy should be initiated as soon as possible after disease evaluation, including MRD assessment (whenever feasible), especially in patients with MRD-positive AML before or after allo-SCT, provided there is adequate hematologic reconstitution. Grade level B-II

- Sorafenib should be transiently discontinued in the case of $\mathrm{GvHD}$ requiring systemic treatment with corticosteroids, but may be cautiously resumed once remission of GvHD is documented. Grade level B-III

- If choosing sorafenib, the recommended post-transplant maintenance dose is $400 \mathrm{mg} /$ day in two divided doses. Patients with MRD-positive disease may receive $800 \mathrm{mg} /$ day in two divided doses, to be adapted according to tolerance. Grade level B-III

- One potential challenge is the lack of approval of sorafenib for AML and its off-label use may not be reimbursed in many/most countries. Ongoing studies will determine the role and modalities of use of midostaurin, gilteritinib or other FLT3 inhibitors in this setting.

- The duration of maintenance therapy is not firmly established, but a minimum of 2 years is recommended, depending on tolerance. Grade level B-III
- Monitoring is recommended for potential drug-drug interactions and long-term side effects.

\section{Aspects to be resolved}

- Standardization of FLT3-ITD allelic ratio in terms of technique and cut-off level

- Indication for allo-SCT in patients with FLT3-ITD AML who belong to the ELN intermediate risk group (high allelic ratio $\geq 0.5$ with concomitant NPM1 mutation) and who achieve MRD negativity.

- Time of withdrawal of immunosuppression

- Pre-emptive versus prophylactic donor lymphocyte infusion

- Post-transplant maintenance with FLT3 inhibitors outside FLT3-ITD AML (immunomodulatory and off-target effects)

- Impact of post-transplant maintenance therapy on immune reconstitution and environment

- Combination of post-transplant FLT3 inhibitors with other drugs such as hypomethylating agents

- Monitoring of patients receiving post-transplant FLT3 inhibitors for potential extramedullary relapse or aggressive clone selection.

\section{References}

1. Gilliland DG, Griffin JD. The roles of FLT3 in hematopoiesis and leukemia. Blood. 2002;100(5):1532-1542

2. Griffith J, Black J, Faerman C, et al. The structural basis for autoinhibition of FLT3 by the juxtamembrane domain. Mol Cell. 2004;13(2):169-178.

3. Mizuki M, Fenski $\mathrm{R}$, Halfter $\mathrm{H}$, et al. Flt3 mutations from patients with acute myeloid leukemia induce transformation of $32 \mathrm{D}$ cells mediated by the Ras and STAT5 pathways. Blood. 2000;96(12):3907-3914

4. Hayakawa F, Towatari M, Kiyoi H, et al. Tandem-duplicated Flt3 constitutively activates STAT5 and MAP kinase and introduces autonomous cell growth in IL-3dependent cell lines. Oncogene. 2000;19(5): 624-631.

5. Roux PP, Blenis J. ERK and p38 MAPK-activated protein kinases: a family of protein kinases with diverse biological functions. Microbiol Mol Biol Rev. 2004;68(2):320-344.

6. Manning BD, Cantley LC. AKT/PKB signaling: navigating downstream. Cell. 2007;129(7):1261-1274.

7. Weisberg E, Roesel J, Furet $\mathrm{P}$, et al. Antileukemic effects of novel first- and second-generation FLT3 inhibitors: structureaffinity comparison. Genes Cancer. 2010;1(10):1021-1032.

8. Papaemmanuil E, Gerstung M, Bullinger L, et al. Genomic classification and prognosis in acute myeloid leukemia. $\mathrm{N}$ Engl J Med. 2016;374(23):2209-2221.

9. Cancer Genome Atlas Research Network; Ley TJ, Miller C, Ding L, et al. Genomic and epigenomic landscapes of adult de novo acute myeloid leukemia. N Engl J Med. 2013;368(22):2059-2074.

10. Patel JP, Gonen M, Figueroa ME, et al.
Prognostic relevance of integrated genetic profiling in acute myeloid leukemia. $\mathrm{N}$ Eng J Med. 2012;366(12):1079-1089.

11. Thiede C, Steudel C, Mohr B, et al. Analysis of FLT3-activating mutations in 979 patients with acute myelogenous leukemia: association with FAB subtypes and identification of subgroups with poor prognosis. Blood. 2002;99(12):4326-4335

12. Kottaridis PD, Gale RE, Frew ME, et al. The presence of a FLT3 internal tandem duplication in patients with acute myeloid leukemia (AML) adds important prognostic information to cytogenetic risk group and response to the first cycle of chemotherapy: analysis of 854 patients from the United Kingdom Medical Research Council AML 10 and 12 trials. Blood. 2001;98(6):17521759.

13. Nagel G, Weber D, Fromm E, et al. Epidemiological, genetic, and clinical characterization by age of newly diagnosed acute myeloid leukemia based on an academic population-based registry study (AMLSC BiO). Ann Hematol. 2017;96(12):1993-2003.

14. Nakao M, Yokota $S$, Iwai $T$, et al. Interna tandem duplication of the flt3 gene found in acute myeloid leukemia. Leukemia. 1996;10(12):1911-1918.

15. Yamamoto $Y$, Kiyoi H, Nakano $Y$, et al Activating mutation of D835 within the activation loop of FLT3 in human hematologic malignancies. Blood. 2001;97(8):24342439.

16. Schlenk RF, Dohner K, Krauter J, et al. Mutations and treatment outcome in cytogenetically normal acute myeloid leukemia. N Engl J Med. 2008;358(18):1909-1918.

17. Schlenk RF, Kayser S, Bullinger L, et al. Differential impact of allelic ratio and insertion site in FLT3-ITD-positive AML with respect to allogeneic transplantation. Blood. 2014;124(23):3441-3449.

18. Tallman MS, Wang ES, Altman JK, et al
Acute myeloid leukemia, version 3. 2019, NCCN clinical practice guidelines in oncology. J Natl Compr Canc Netw. 2019;17(6) 721-749.

19. Döhner H, Estey E, Grimwade D, et al Diagnosis and management of AML in adults: 2017 ELN recommendations from an international expert panel. Blood. 2017;129 (4):424-447

20. Garg M, Nagata Y, Kanojia D, et al. Profiling of somatic mutations in acute myeloid leukemia with FLT3-ITD at diagnosis and relapse. Blood. 2015;126(22):2491-2501.

21. Thiede C, Koch S, Creutzig E, et al Prevalence and prognostic impact of NPM1 mutations in 1485 adult patients with acute myeloid leukemia (AML). Blood. 2006;107 (10):4011-4020

22. Pratcorona $M$, Brunet $S$, Nomdedeu J, et al Favorable outcome of patients with acute myeloid leukemia harboring a low-allelic burden FLT3-ITD mutation and concomitant NPM1 mutation: relevance to postremission therapy. Blood. 2013;121(14) 2734-2738.

23. Thiede C, Prior TW, Lavorgna S, et al. FLT3 mutation assay laboratory cross validation: results from the CALGB 10603/ratify trial in patients with newly diagnosed FLT3-mutated scute myeloid leukemia (AML). Blood. 2018;132(Suppl 1):2800

24. Kiyoi H, Naoe T, Nakano Y, et al. Prognostic implication of FLT3 and N-RAS gene mutations in acute myeloid leukemia. Blood. 1999;93(9):3074-3080.

25. Yanada M, Matsuo K, Suzuki T, Kiyoi $H$ Naoe T. Prognostic significance of FLT3 internal tandem duplication and tyrosine kinase domain mutations for acute myeloid leukemia: a meta-analysis. Leukemia. 2005;19(8):1345-1349.

26. Whitman SP, Archer KJ, Feng L, et al Absence of the wild-type allele predicts poor prognosis in adult de novo acute myeloid 
leukemia with normal cytogenetics and the internal tandem duplication of FLT3: a Cancer and Leukemia Group B study. Cancer Res. 2001;61(19):7233-7239.

27. Frohling S, Schlenk RF, Breitruck J, et al. Prognostic significance of activating FLT3 mutations in younger adults (16 to 60 years) with acute myeloid leukemia and normal cytogenetics: a study of the AML Study Group Ulm. Blood. 2002;100(13):4372-4380.

28. Kayser S, Schlenk RF, Londono MC, et al. Insertion of FLT3 internal tandem duplication in the tyrosine kinase domain-1 is associated with resistance to chemotherapy and inferior outcome. Blood. 2009;114(12):23862392.

29. Mead AJ, Linch DC, Hills RK, Wheatley K, Burnett AK, Gale RE. FLT3 tyrosine kinase domain mutations are biologically distinct from and have a significantly more favorable prognosis than FLT3 internal tandem duplications in patients with acute myeloid leukemia. Blood. 2007;110(4):1262-1270.

30. Whitman SP, Ruppert AS, Radmacher MD, et al. FLT3 D835/1836 mutations are associated with poor disease-free survival and a distinct gene-expression signature among younger adults with de novo cytogenetically normal acute myeloid leukemia lacking FLT3 internal tandem duplications. Blood. 2008;111(3):1552-1559.

31. Moreno I, Martin G, Bolufer P, et al. Incidence and prognostic value of FLT3 internal tandem duplication and D835 mutations in acute myeloid leukemia. Haematologica. 2003;88(1):19-24

32. Bacher U, Haferlach C, Kern W, Haferlach T, Schnittger S. Prognostic relevance of FLT3TKD mutations in AML: the combination matters--an analysis of 3082 patients. Blood. 2008;111(5):2527-2537.

33. Voso MT, Larson RA, Prior T, et al. Ratify (Alliance 10603): prognostic impact of FLT3 tyrosine kinase domain (TKD) and NPM1 mutation status in patients with newly diagnosed acute myeloid leukemia (AML) treated with midostaurin or placebo plus standard chemotherapy. Blood. 2018;132 (Suppl 1):2668.

34. Boddu P, Kantarjian H, Borthakur G, et al. Co-occurrence of FLT3-TKD and NPM1 mutations defines a highly favorable prognostic AML group. Blood Adv. 2017;1 (19):1546-1550.

35. Perry M, Bertoli S, Rocher C, et al. FLT3TKD mutations associated with NPM1 mutations define a favorable-risk group in patients with acute myeloid leukemia. Clin Lymphoma Myeloma Leuk. 2018;18(12): e545-e550.

36. Short NJ, Kantariian H, Ravandi F, Daver N. Emerging treatment paradigms with FLT3 inhibitors in acute myeloid leukemia. Ther Adv Hematol. 2019;10:2040620719827310.

37. Stone RM, Mandrekar SJ, Sanford BL, et al. Midostaurin plus chemotherapy for acute myeloid leukemia with a FLT3 mutation. N Engl J Med. 2017;377(5):454-464.

38. Perl AE, Martinelli G, Cortes JE, et al. Gilteritinib or chemotherapy for relapsed or refractory FLT3-mutated AML. N Engl J Med. 2019;381(18):1728-1740.

39. Cortes JE, Khaled S, Martinelli G, et al. Quizartinib versus salvage chemotherapy in relapsed or refractory FLT3-ITD acute myeloid leukaemia (QuANTUM-R): a multicentre, randomised, controlled, open-label, phase 3 trial. Lancet Oncol. 2019;20(7):984997.

40. Wang ES, Tallman MS, Stone RM, et al. Low relapse rate in younger patients $\leq 60$ years old with newly diagnosed FLT3-mutated acute myeloid leukemia (AML) treated with crenolanib and cytarabine/anthracycline chemotherapy. Blood. 2017;130(Suppl 1):566.

41. Rollig C, Serve H, Huttmann A, et al. Addition of sorafenib versus placebo to standard therapy in patients aged 60 years or younger with newly diagnosed acute myeloid leukaemia (SORAML): a multicentre, phase 2, randomised controlled trial. Lancet Oncol. 2015;16(16):1691-1699.

42. Serve H, Krug U, Wagner R, et al. Sorafenib in combination with intensive chemotherapy in elderly patients with acute myeloid leukemia: results from a randomized, placebo-controlled trial. J Clin Oncol. 2013;31 (25):3110-3118

43. Antar A, Otrock ZK, El-Cheikh J, et al Inhibition of FLT3 in AML: a focus on sorafenib. Bone Marrow Transplant. 2017;52(3):344-351

44. Macdonald DA, Assouline SE, Brandwein J, et al. A phase I/II study of sorafenib in combination with low dose cytarabine in elderly patients with acute myeloid leukemia or high-risk myelodysplastic syndrome from the National Cancer Institute of Canada Clinical Trials Group: trial IND.186. Leuk Lymphoma. 2013;54(4):760-766.

45. Borthakur $G$, Kantariian $H$, Ravandi F, et al Phase I study of sorafenib in patients with refractory or relapsed acute leukemias. Haematologica. 2011;96(1):62-68

46. Metzelder SK, Schroeder T, Lubbert M, et al. Long-term survival of sorafenib-treated FLT3-ITD-positive acute myeloid leukaemia patients relapsing after allogeneic stem cell transplantation. Eur J Cancer. 2017;86:233239.

47. Cortes JE, Kantarjian H, Foran JM, et al. Phase I study of quizartinib administered daily to patients with relapsed or refractory acute myeloid leukemia irrespective of FMS like tyrosine kinase 3-internal tandem duplication status. I Clin Oncol. 2013;31(29): 3681-3687

48. Levis MJ, Perl AE, Altman JK, et al. Results of a first-in-human, phase I/II trial of ASP2215, a selective, potent inhibitor of FLT3/Axl in patients with relapsed or refractory (R/R) acute myeloid leukemia (AML). I Clin Oncol. 2015;33(15_suppl):7003

49. Leung AY, Man CH, Kwong YL. FLT3 inhibition: a moving and evolving target in acute myeloid leukaemia. Leukemia. 2013;27(2): 260-268.

50. Thol F, Schlenk RF, Heuser M, Ganser A. How I treat refractory and early relapsed acute myeloid leukemia. Blood. 2015;126(3) 319-327.

51. Winkler J, Rech D, Kallert S, et al. Sorafenib induces sustained molecular remission in FLT3-ITD positive AML with relapse after second allogeneic stem cell transplantation without exacerbation of acute GVHD: a case report. Leuk Res. 2010;34(10):e270-272.

52. Sharma M, Ravandi F, Bayraktar UD, et al. Treatment of FLT3-ITD-positive acute myeloid leukemia relapsing after allogeneic stem cell transplantation with sorafenib. Biol Blood Marrow Transplant. 2011;17(12): 1874-1877.

53. Metzelder SK, Schroeder T, Finck A, et al. High activity of sorafenib in FLT3-ITD-positive acute myeloid leukemia synergizes with allo-immune effects to induce sustained responses. Leukemia. 2012;26(11):23532359

54. Rautenberg C, Nachtkamp K, Dienst A, et al. Sorafenib and azacitidine as salvage ther- apy for relapse of FLT3-ITD mutated AML after allo-SCT. Eur J Haematol. 2017;98(4): 348-354.

55. Bazarbachi A, Labopin M, Battipaglia G, et al. Sorafenib improves survival of FLT3mutated acute myeloid leukemia in relapse after allogeneic stem cell transplantation: a report of EBMT Acute Leukemia Working Party. Haematologica. 2019;104(9):e398e401.

56. Ravandi F, Alattar ML, Grunwald MR, et al. Phase 2 study of azacytidine plus sorafenib in patients with acute myeloid leukemia and FLT-3 internal tandem duplication mutation. Blood. 2013;121(23):4655-4662.

57. Sid S, Rey J, Charbonnier A, et al. Treatment of post-transplant relapse of FLT3-ITD mutated AML using 5-azacytidine and sorafenib bitherapy. Clin Lymphoma Myeloma Leuk. 2017;17(4):241-242.

58. Sasaki K, Kantarjian HM, Kadia T, et al. Sorafenib plus intensive chemotherapy improves survival in patients with newly diagnosed, FLT3-internal tandem duplication mutation-positive acute myeloid leukemia. Cancer. 2019;125(21):3755-3766.

59. Yalniz F, Abou Dalle I, Kantarjian H, et al Prognostic significance of baseline FLT3-ITD mutant allele level in acute myeloid leukemia treated with intensive chemotherapy with/without sorafenib. Am J Hematol. 2019;94(9):984-991

60. Battipaglia G, Ruggeri A, Massoud R, et al Efficacy and feasibility of sorafenib as a maintenance agent after allogeneic hematopoietic stem cell transplantation for Fms-like tyrosine kinase 3-mutated acute myeloid leukemia. Cancer. 2017;123(15): 2867-2874.

61. Chen YB, Li S, Lane AA, et al. Phase I trial of maintenance sorafenib after allogeneic hematopoietic stem cell transplantation for fms-like tyrosine kinase 3 internal tandem duplication acute myeloid leukemia. Biol Blood Marrow Transplant. 2014;20(12):2 042-2048.

62. Brunner AM, Li S, Fathi AT, et al Haematopoietic cell transplantation with and without sorafenib maintenance for patients with FLT3-ITD acute myeloid leukaemia in first complete remission. Br J Haematol. 2016;175(3):496-504.

63. Bazarbachi A, Labopin M, Battipaglia G, et al. Allogeneic stem cell transplantation for FLT3-mutated acute myeloid leukemia: in vivo T-cell depletion and posttransplant sorafenib maintenance improve survival. A retrospective acute Leukemia Working Party-European Society for Blood and Marrow Transplant Study. Clin Hematol Int. 2019:1(1):58-74

64. Burchert A. Sorafenib As Maintenance therapy post allogeneic stem cell transplantation for FLT3-ITD positive AML: results from the randomized, double-blind, placebo-controlled multicentre sormain trial. Blood. 2018:132(Suppl 1):661.

65. Battipaglia G, Massoud R, Ahmed SO, et al. Efficacy and feasibility of sorafenib as a maintenance agent after allogeneic hematopoietic stem cell transplantation for Fms-like tyrosine kinase 3 mutated acute myeloid leukemia: an update. Clin Lymphoma Myeloma Leuk. 2019;19(8):506508.

66. Bornhauser $M$, Illmer $T$, Schaich $M$, et al. Improved outcome after stem-cell transplantation in FLT3/ITD-positive AML. Blood. 2007:109(5):2264-2265; author reply 2265.

67. Gale RE, Hills R, Kottaridis PD, et al. No evidence that FLT3 status should be considered 
EBMT ALWP recommendation for allo-SCT for FLT3 AML

as an indicator for transplantation in acute myeloid leukemia (AML): an analysis of 1135 patients, excluding acute promyelocytic leukemia, from the UK MRC AML10 and 12 trials. Blood. 2005;106(10):3658-3665.

68. Meshinchi S, Arceci RJ, Sanders JE, et al. Role of allogeneic stem cell transplantation in FLT3/ITD-positive AML. Blood. 2006;108(1):400; author reply 400-401.

69. DeZern AE, Sung A, Kim S, et al. Role of allogeneic transplantation for FLT3/ITD acute myeloid leukemia: outcomes from 133 consecutive newly diagnosed patients from a single institution. Biol Blood Marrow Transplant. 2011;17(9):1404-1409.

70. Sengsayadeth SM, Jagasia M, Engelhardt $\mathrm{BG}$, et al. Allo-SCT for high-risk AML-CR1 in the molecular era: impact of FLT3/ITD outweighs the conventional markers. Bone Marrow Transplant. 2012;47(12):1535-1537.

71. Kayser S DK, Krauter J, Kohne C, et al. Allogeneic transplantation from matched related and unrelated donors in first complate remission in younger adult $\mathrm{AML}$ patients with FLT3 internal tandem duplicaions. Bone Marrow Transplant. 2011;18(6):395-400.

72. Hemmati P TT, Vuong LG, le Coutre PD, Dorken B, Arnold R. Allogeneic stem cell transplantation for cytogenetically normal acute myeloid leukemia: impact of FLT3 and NPM1 mutational status. Blood. 2013; 122(21):2104

73. Liegel J, Courville E, Sachs Z, Ustun C. Use of sorafenib for post-transplant relapse in FLT3/ITD-positive acute myelogenous leukemia: maturation induction and cytotoxic effect. Haematological. 2014;99(11): e222-224.

74. Deal A, Sengsayadeth S, An KW, et al. Does FLT3 mutation impact survival after hematopoietic stem cell transplantation for acute myeloid leukemia? A Center for International Blood and Marrow Transplant Research (CIBMTR) analysis. Cancer. 2016;122(19):3005-3014.

75. DeZern AE, Sung A, Kim S, et al. Patients with FLT3/ITD AML may benefit from allogenic transplant in first remission: outcomes from a consecutive series of patients at a single institution. Blood. 2010;116 (21):2172.

76. Ivy A, Hills RK, Simpson MA, et al. Assessment of minimal residual disease in standard-risk AML. N Engl J Med. 2016;374 (5):422-433.

77. Terwijn M, van Putter WLJ, Keller A, et al. High prognostic impact of flow cytometric minimal residual disease detection in acute myeloid leukemia: data from the HOVON/SAKK AML 42A study. J Chin Oncol. 2013;31(31):3889-3897.

78. Schuurhuis GJ, Heuser M, Freeman S, et al. Minimal/measurable residual disease in AML: a consensus document from the European LeukemiaNet MRD Working Party. Blood. 2018;131(12):1275-1291.

79. Chang Y-J, Huang X-J. Haploidentical stem cell transplantation: anti-thymocyte globslin-based experience. Serin Hematol. 2016;53(2):82-89

80. McCurdy SR, Kasamon YL, Kanakry CG, et al. Comparable composite endpoints after HLA-matched and HLA-haploidentical transplantation with post-transplantation cyclophosphamide. Haematological. 2017; 102(2):391-400.

81. Chang Y-Y, Mo X-D, Zhang X-H, et al. FLT3 internal tandem duplication does not impact prognosis after haploidentical allogeneic hematopoietic stem cell transplantation in
AML patients. Bone Marrow Transplant. 2019;54(9):1462-1470.

82. Schiller GJ, Turtle P, Desai P. Allogeneic hematopoietic stem cell transplantation in FLT3-ITD-positive acute myelogenous leukemia: the role for FLT3 tyrosine kinase inhibitors post-transplantation. Biol Blood Marrow Transplant. 2016;22(6):982-990.

83. Shouval R, Fin JA, Labopin M, et al. Outcomes of allogeneic haematopoietic stem cell transplantation from HLAmatched and alternative donors: a European Society for Blood and Marrow Transplantation registry retrospective analysis. Lancet Haematol. 2019;6(11):e573-e584.

84. Yuan L, Wang Y, Huang F, et al. Effect of sorafenib on the outcomes of patients with FLT3 ITD acute myeloid leukemia undergoing allogeneic hematopoietic stem cell transplantation. Cancer. 2018;124(9):1954-1963.

85. Bazarbachi AH, Al tamed R, Malard F, Monty M, Bazarbachi A. Allogeneic transplant for FLT3-ITD mutated AML: a focus on FLT3 inhibitors before, during, and after transplant. Then Adv Hematol. 2019;10: 2040620719882666.

86. Williams PL, Webb C. The Delphi technique: a methodological discussion. J Adv Nurse. 1994;19(1):180-186.

87. Couriel DR. Ancillary and supportive care in chronic graft-versus-host disease. Best Pract Res Chin Haematol. 2008;21(2):291-307.

88. Ho $\mathrm{AD}$, Schetelig J, Bochtler $\mathrm{T}$, et al. Allogeneic stem cell transplantation improves survival in patients with acute myeloid leukemia characterized by a high allelic ratio of mutant FLT3-ITD. Biol Blood Marrow Transplant. 2016;22(3):462-469.

89. Versluis J, In 't Hour FE, Devillier R, et al. Comparative value of post-remission treatmont in cytogenetically normal AML subclassified by NPM1 and FLT3-ITD allelic ratio. Leukemia. 2017;31(1):26-33.

90. Rombouts WJ, Blokland I, Lowenberg B, Ploemacher RE. Biological characteristics and prognosis of adult acute myeloid leukemia with internal tandem duplications in the Flt gene. Leukemia. 2000;14(4):675683.

91. Döhner K, Thiede C, John N, et al. Impact of NPM1/FLT3-ITD genotypes defined by the 2017 European LeukemiaNet in patients with acute myeloid leukemia. Blood. 2020;135(5):371-380.

92. Brunet S, Labopin M, Esteve J, et al. Impact of FLT3 internal tandem duplication on the outcome of related and unrelated hematopoietic transplantation for adult acute myeloid leukemia in first remission: a retrospective analysis. J Chin Oncol. 2012;30(7):735-741.

93. Ma Y, Wu Y, Then Z, Chang X, Zing D, Kong P. Is allogeneic transplantation really the best treatment for FLT3/ITD-positive acute myeloid leukemia? A systematic review. Chin Transplant. 2015;29(2):149-160.

94. Doubek M, Muzik J, Szotkowski T, et al. Is FLT3 internal tandem duplication significant indicator for allogeneic transplantation in acute myeloid leukemia? An analysis of patients from the Czech Acute Leukemia Clinical Register (ALERT). Neoplasma. 2007;54(1):89-94.

95. Lin PH, Lin CC, Yang HI, et al. Prognostic impact of allogeneic hematopoietic stem cell transplantation for acute myeloid leukemia patients with internal tandem duplication of FLT3. Leuk Res. 2013;37(3):287-292.

96. Canaan J, Labopin M, Huang XJ, et al. T-cell replete haploidentical stem cell transplantaion attenuates the prognostic impact of

FLT3-ITD in acute myeloid leukemia: a report from the Acute Leukemia Working Party of the European Society for Blood and Marrow Transplantation. Am J Hematol. 2018;93(6):736-744.

97. Schmid C, Labopin M, Socie G, et al. Outcome of patients with distinct molecular genotypes and cytogenetically normal AML after allogeneic transplantation. Blood. 2015;126(17):2062-2069.

98. Thanarajasingam G, Kim HT, Cutler C, et al. Outcome and prognostic factors for patients who relapse after allogeneic hematopoietic stem cell transplantation. Biol Blood Marrow Transplant. 2013;19(12):1713-1718.

99. Sakaguchi M, Yamaguchi H, Najima Y, et al. Prognostic impact of low allelic ratio FLT3ITD and NPM1 mutation in acute myeloid leukemia. Blood Adv. 2018;2(20):2744-2754.

100. Oran B, Cortes J, Beitinjaneh A, et al. Allogeneic transplantation in first remission improves outcomes irrespective of FLT3ITD allelic ratio in FLT3-ITD-positive acute myelogenous leukemia. Biol Blood Marrow Transplant. 2016;22(7):1218-1226.

101. Zhao X, Wang Z, Run G, et al. Impact of pre-transplantation minimal residual disease determined by multiparameter flow cytontry on the outcome of AML patients with FLT3-ITD after allogeneic stem cell transplantation. Ann Hematol. 2018;97(6):967975.

102. Popescu B, Chela S, Thompson J, et al. Timed sequential salvage chemotherapy for relapsed or refractory acute myeloid leukemia. Chin Hematol Int. 2019;2(1):27-31.

103. Pore X, Labopin M, Martens J, et al. Allogeneic stem cell transplantation in adult patients with acute myeloid leukaemia and $17 p$ abnormalities in first complete remssion: a study from the Acute Leukemia Working Party (ALWP) of the European Society for Blood and Marrow Transplantation (EBMT). J Hematol Oncol. 2017;10(1):20

104. Labour G, Dulucq S, Labopin M, et al. Potent graft-versus-leukemia effect after reduced-intensity allogeneic SCT for intermediate-risk AML with FLT3-ITD or wildtype NPM1 and CEBPA without FLT3-ITD. Biol Blood Marrow Transplant. 2012;18(12): 1845-1850.

105. Gale RE, Green C, Allen C, et al. The impact of FLT3 internal tandem duplication mutant level, number, size, and interaction with NPM1 mutations in a large cohort of young adult patients with acute myeloid leukemia. Blood. 2008;111(5):2776-2784

106. Cabala S, Saliva R, Oran B, et al. Relapse risk and survival in patients with FLT3 mutated acute myeloid leukemia undergoing stem cell transplantation. Am J Hematol. 2017;92(4):331-337.

107. Kayser S, Bonner A, Thiede C, et al. Pretransplant NPM1 MRD levels predict outcome after allogeneic hematopoietic stem cell transplantation in patients with acute myeloid leukemia. Blood Cancer J. 2016;6(7):e449-e449.

108. Pfeiffer T, Schleuning M, Mayer J, et al. Influence of molecular subgroups on outcome of acute myeloid leukemia with normall karyotype in 141 patients undergoing salvage allogeneic stem cell transplantation in primary induction failure or beyond first relapse. Haematological. 2013;98(4):518525.

109. Grin NC, Labopin M, Braise D, et al. Stem cell transplantation from a haploidentical donor versus a genoidentical sister for adult male patients with acute myelogenous 
leukemia in first remission: a retrospective study from the Acute Leukemia Working Party of the European Society for Blood and Marrow Transplantation. Cancer. 2019;126 (5):1004-1015.

110. Versluis J, Labopin M, Ruggeri A, et al. Alternative donors for allogeneic hematopoietic stem cell transplantation in poor-risk AML in CR1. Blood Adv. 2017:1(7):477-485

111. Kharfan-Dabaja MA, Labopin M, Polge E, et al. Association of second allogeneic hematopoietic cell transplant vs donor lymphocyte infusion with overall survival in patients with acute myeloid leukemia relapse. JAMA Oncol. 2018;4(9):1245-1253.

112. Levis MJ, Chen Y-B, Hamadani M, et al. FLT3 inhibitor maintenance after allogeneic transplantation: is a placebo-controlled, randomized trial ethical? J Clin Oncol. 2019; 37(19):1604-1607.

113. Stone RM, Mandrekar SJ, Sanford BL, et al. The addition of midostaurin to standard chemotherapy decreases cumulative incidence of relapse (CIR) in the international prospective randomized, placebo-controlled, double-blind trial (CALGB 10603 /
RATIFY [Alliance]) for newly diagnosed acute myeloid leukemia (AML) Patients with FLT3 mutations. Blood. 2017;130 (Suppl 1):2580.

114. Maziarz RTT, Patnaik MM, Scott BL, et al. Radius: a phase 2 randomized trial investigating standard of care \pm midostaurin after allogeneic stem cell transplant in FLT3-ITDmutated AML. Blood. 2018;132(Suppl 1):662.

115. Schlenk RF, Weber D, Fiedler W, et al Midostaurin added to chemotherapy and continued single-agent maintenance therapy in acute myeloid leukemia with FLT3-ITD. Blood. 2019;133(8):840-851.

116. Antar A, Kharfan-Dabaja MA, Mahfouz R, Bazarbachi A. Sorafenib maintenance appears safe and improves clinical outcomes in FLT3-ITD acute myeloid leukemia after allogeneic hematopoietic cell transplantation. Clin Lymphoma Myeloma Leuk. 2015;15(5):298-302.

117. Tschan-Plessl A, Halter JP, Heim D, Medinger M, Passweg JR, Gerull S. Synergistic effect of sorafenib and cGvHD in patients with high-risk FLT3-ITD+AML allows long-term disease control after allo- geneic transplantation. Ann Hematol. 2015;94(11):1899-1905

118. Yokoyama H, Lundqvist A, Su S, Childs R Toxic effects of sorafenib when given early after allogeneic hematopoietic stem cell transplantation. Blood. 2010;116(15):28582859.

119. Mathew NR, Baumgartner F, Braun L, et al. Sorafenib promotes graft-versus-leukemia activity in mice and humans through IL-15 production in FLT3-ITD-mutant leukemia cells. Nat Med. 2018;24(3):282-291.

120.Levis MJ, Hamadani M, Logan B, et al. A phase 3 , trial of gilteritinib, as maintenance therapy after allogeneic hematopoietic stem cell transplantation in patients with FLT3ITD+ AML. J Clin Oncol. 2018;36 (15_Suppl):TPS7075.

121. Canaani J. Management of AML Beyond "3+ 7" in 2019. Clin Hematol Int. 2019;1(1): 10-18.

122. Culos K, Byrne M. Salvage therapy after allogeneic hematopoietic cell transplantation: targeted and low-intensity treatment options in myelodysplastic syndrome and acute myeloid leukemia. Clin Hematol Int. 2019;1(2):94-100 